\title{
Uma revisão integrativa sobre o uso de plantas aromáticas encontradas na Amazônia
} na promoção da fitoterapia

An integrative review on the use of aromatic plants found in the Amazon to promote herbal medicine

Una revisión integradora sobre el uso de plantas aromáticas que se encuentran en la Amazonía para promover la medicina herbal

Recebido: 25/10/2021 | Revisado: 01/11/2021 | Aceito: 04/11/2021 | Publicado: 07/11/2021

Lucilane Maria Penaforth Ramos ORCID: https://orcid.org/0000-0001-8629-000X Universidade Nilton Lins, Brasil E-mail: lucilane36@gmail.com

Gabriel Oliveira de Souza

ORCID: https://orcid.org/0000-0001-9168-074X Universidade Nilton Lins, Brasil

E-mail: gabriel.souza@uniniltonlins.edu.br

\begin{abstract}
Resumo
Devido as suas propriedades terapêuticas, as plantas aromáticas são bastante utilizadas pela população para fins medicinais, aromatizantes, condimentares e para estudos científicos a respeito das ações farmacológicas: antiinflamatória, antiespasmódica, antioxidantes, antissépticas, antimicrobianas, calmantes, carminativa, entre outras. Por isso, o objetivo vem com o intuito de facilitar a produção das plantas aromáticas para auxiliar a população e os profissionais da saúde a buscar conhecimentos sobre os efeitos farmacológicos e fisiológicos dos compostos bioativos de cada planta em prol da saúde humana, através da fitoterapia. O trabalho foi embasado em pesquisas de artigos publicados no período de 2013 a 2021 de acordo com a revisão bibliográfica nas plataformas cientificas: SciELO e PUBMED, com respectivas seleções e leituras dos artigos no mês de julho a setembro. No total foram obtidos 30 artigos, nos quais foram excluídos aqueles que continham repetições e não apresentavam contexto compatível com o tema, restando apenas 21 artigos selecionados. Com base, na revisão bibliográfica foram selecionadas 10 espécies de plantas aromáticas (Alecrim; Orégano; Boldo brasileiro; Arruda; Hortelã comum; Erva-cidreira; Capim-limão; Alfavaca; Sálvia; Alfazema), com suas respectivas informações: nome cientifico, nome popular, parte usada da planta, propriedade farmacológica, indicação terapêutica. O estudo identificou dentro da fitoterapia e da medicinal tradicional, diferentes abordagens e costumes acerca do uso correto no manejo de plantas medicinais. Além de contribuir para difundir o conhecimento a cerca dessa classe de plantas (aromáticas), o presente estudo fornece ferramentas com base na leitura acerca da forma do uso para o preparo mais eficiente dessas espécies aqui revisadas.
\end{abstract}

Palavras-chave: Plantas aromáticas; Atividade farmacológica; Fitoterapia.

\begin{abstract}
Due to their therapeutic properties, aromatic plants are widely used by the population for medicinal, flavoring, flavoring and scientific studies regarding pharmacological actions: anti-inflammatory, antispasmodic, antioxidant, antiseptic, antimicrobial, calming, carminative, among others. Therefore, the objective is to facilitate the production of aromatic plants to help the population and health professionals to seek knowledge about the pharmacological and physiological effects of the bioactive compounds of each plant in favor of human health, through herbal medicine. The work was based on research of articles published in the period from 2013 to 2021, according to the literature review in scientific platforms: SciELO and PUBMED, with respective selections and readings of articles from July to September. In total, 30 articles were obtained, from which those that contained repetitions and did not present a context compatible with the theme were excluded, leaving only 21 selected articles. Based on the bibliographical review, 10 species of aromatic plants were selected (Rosecrim; Oregano; Brazilian Boldo; Arruda; Common Mint; Lemongrass; Lemongrass; Alfavaca; Sage; Lavender), with their respective information: scientific name, name popular, used part of the plant, pharmacological property, therapeutic indication. The study identified, within phytotherapy and traditional medicine, different approaches and customs regarding the correct use in the management of medicinal plants. In addition to contributing to the dissemination of knowledge about this class of (aromatic) plants, the present study provides tools based on reading about how to use them for the most efficient preparation of these species reviewed here.
\end{abstract}

Keywords: Aromatic plants; Pharmacological activity; Phytotherapy. 


\begin{abstract}
Resumen
Debido a sus propiedades terapéuticas, las plantas aromáticas son ampliamente utilizadas por la población con fines medicinales, aromatizantes, aromatizantes y científicos sobre acciones farmacológicas: antiinflamatorias, antiespasmódicas, antioxidantes, antisépticas, antimicrobianas, calmantes, carminativas, entre otras. Por tanto, el objetivo es facilitar la producción de plantas aromáticas para ayudar a la población y a los profesionales de la salud a buscar conocimiento sobre los efectos farmacológicos y fisiológicos de los compuestos bioactivos de cada planta a favor de la salud humana, a través de la fitoterapia. El trabajo se basó en la investigación de artículos publicados en el período de 2013 a 2021, de acuerdo con la revisión de literatura en plataformas científicas: SciELO y PUBMED, con respectivas selecciones y lecturas de artículos de julio a septiembre. En total se obtuvieron 30 artículos, de los cuales se excluyeron aquellos que contenían repeticiones y no presentaban un contexto compatible con la temática, quedando solo 21 artículos seleccionados. Con base en la revisión bibliográfica, se seleccionaron 10 especies de plantas aromáticas (Rosecrim; Orégano; Boldo brasileño; Arruda; Menta común; Limoncillo; Limoncillo; Alfavaca; Salvia; Lavanda), con sus respectivas informaciones: nombre científico, nombre popular, parte usada de la planta, propiedades farmacológicas, indicación terapéutica. El estudio identificó, dentro de la fitoterapia y la medicina tradicional, diferentes enfoques y costumbres en cuanto al uso correcto en el manejo de las plantas medicinales. Además de contribuir a la difusión del conocimiento sobre esta clase de plantas (aromáticas), El presente estudio proporciona herramientas basadas en la lectura sobre cómo usarlas para la preparación más eficiente de estas especies aquí reseñadas.
\end{abstract}

Palabras clave: Plantas aromáticas; Actividad farmacológica; Fitoterapia.

\title{
1. Introdução
}

Plantas aromáticas são espécies vegetais, os quais são constituídos por princípios ativos totais ou parcialmente por essências devido ao seu aroma capaz de sensibilizar o olfato de forma agradável (Correa Júnior et al., 2014). Atualmente, são plantas usualmente consumidas e utilizadas para fins nutricionais e medicinais, através de preparações de infusões e decocções realizadas no cotidiano familiar ou comercial, por apresentarem vários benefícios à saúde do consumidor (Ferreira et al., 2017).

Dentre esses benefícios pode-se citar ações anti-inflamatórias, espasmódicas, antioxidantes, antimicrobianos, antissépticos, entre outras. Com isso, surgem fatores que despertam a atenção dos programas de assistência à saúde, por causa da grande diversidade vegetal e facilidade de utilizar estas plantas aromáticas devido ao seu baixo custo. Assim, a população cerca de $80 \%$ utilizam a medicina tradicional fazendo uso de extratos vegetais e plantas medicinais, especificamente na atenção primária à saúde associado à terapêutica, segundo a OMS (Organização Mundial da Saúde) (Ianck, 2017).

Segundo Silva et al. (2018), as plantas aromáticas são consideradas de usos múltiplos por serem utilizadas para vários fins terapêuticos, além de ser utilizada como inseticidas, repelentes e adubos verdes. Além de que, esse crescimento de recursos fitoterápicos ocorre por causa dos componentes produzidos de certas partes especificas das plantas como: folhas, raízes, flores, sementes, cascas entre outros. Logo, obtêm-se de metabólitos e compostos voláteis, principalmente, os óleos essenciais ou voláteis, o mais utilizados pela área farmacêutica, cosmética, alimentícia e industrias de perfumaria (Rodrigues, Santos \& Fortuna, 2020).Esses compostos aromáticos, geralmente líquidos, são responsáveis pelas misturas de vários compostos aromatizantes característicos das plantas aromáticas, incluindo suas propriedades odoríferas e os sabores (Cruz \& Bezerra, 2018).

Por existir vários tipos de espécies de plantas aromáticas na Amazônia, a estimulação racional do uso de plantas medicinais vem fortalecer os conhecimentos sobre os processos farmacológicos e fisiológicos que possam ampliar o entendimento acadêmico, profissional ou familiar em relação a fitoterapia. A utilização destas plantas aromáticas compõe de vários compostos bioativos (fontes naturais), além de suas propriedades aromatizantes, condimentares e medicinais, pois vem sendo usadas desde a antiguidade devido aos benefícios terapêuticos fornecidos à saúde (Monthana et al., 2019).

A produção e o cultivo correto de plantas medicinais têm um papel importante em relação à estudos científicos devido aos compostos voláteis presentes nos óleos essenciais e em extratos obtidos de diferentes partes destas plantas aromáticas, um estudo que pode auxiliar acadêmicos a realizar pesquisas da efetividade de espécies em relação ao desenvolvimento de novos medicamentos fitoterápicos, e também, no combate de infecções ou patologias (Ferreira et al., 2017). 
Com base no crescimento econômico a partir do uso de plantas e seus derivados a utilização de espécies vegetais abrange um mercado altamente promissor quando usado para fins terapêuticos, produção de cosméticos, alimentícios, entre outros, com o intuito de inserir no mercado mais produtos que possam contribuir para o desenvolvimento econômico (Lima, 2018).

Por esses motivos, o artigo cientifico teve como objetivo uma revisão bibliográfica integrativa sobre espécies de plantas aromáticas presentes na Amazônia, selecionando-as com base na literatura, espécies aromáticas presentes na região realizando um levantamento acerca da forma de uso e indicação farmacológica.

\section{Metodologia}

Este é um estudo de revisão de literatura bibliográfica, que busca sintetizar o conteúdo de vários estudos e analisar criticamente as informações obtidas através de uma visão mais atual dos fatos relacionados. Duas plataformas digitais foram utilizadas para a coleta de dados: Scielo e Pubmed no período de julho a setembro de 2021. Como base para essa pesquisa foram selecionados artigos nos anos de 2013 a 2021.

Após triagem, total foram obtidos 30 artigos, sendo 12 oriundos da PUBMED e 18 da Scielo. Foram excluídos aqueles cuja espécies de plantas não apresentavam compostos aromáticos ou não apresentavam temática compatível com o objetivo do tema proposto neste estudo.

Por fim os artigos foram avaliados quando a repetição, aqueles que continham repetições foram excluídos de uma base de dados, assim, 21 artigos corresponderam aos parâmetros recomendados conforme leitura realizada. A seleção ocorreu mediante os principais descritores que foram: Plantas aromáticas do Amazonas, uso de plantas medicinais na fitoterapia, cultivo de plantas aromáticas medicinais. Em seguida, os resultados dos estudos foram sintetizados, procurando cobrir sua relevância e principais objetivos. Os dados dos estudos foram reunidos e sistematizados, caracterizando a análise crítica com o objetivo de gerar novos entendimentos sobre uma correlação entre a compreensão popular e as diversas formas de aplicação do conhecimento científico dessas plantas medicinais na forma de um quadro sinóptico contendo: Nome popular da planta, nome cientifico, partes utilizadas, atividades farmacológicas, indicação terapêuticas e por Autor/Ano. 
Figura 1: Fluxo adotado na revisão bibliográfica integrativa, afim de se obter os artigos para montagem do quadro sinóptico.

Artigos identificados nas

buscas por meio das bases

de dados $(n=30)$

PUBMED, SCIELO

$\downarrow$

Número de artigos após remoção por serem duplicados $(n=26)$

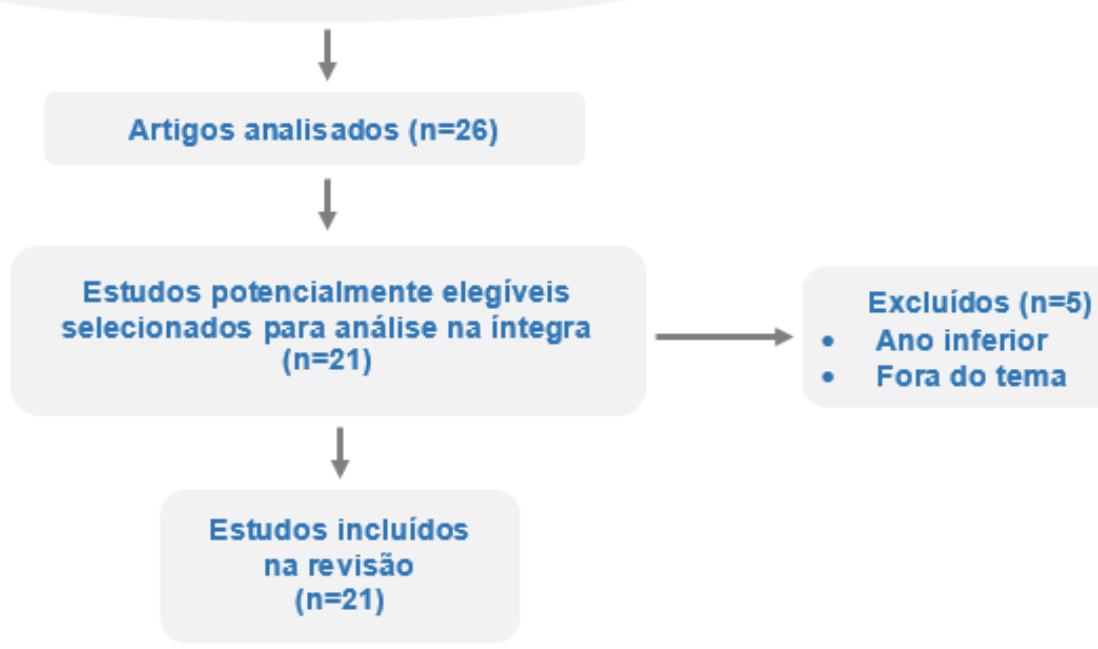

Fonte: Autores.

\section{Resultados e Discussões}

A história da utilização das plantas aromáticas para fins medicinais, cosméticos e no realce dos sabores nos alimentos, vem desde a antiguidade, devido as descobertas de suas propriedades farmacêuticas parcialmente relacionadas aos compostos voláteis (óleos essenciais), e ainda por serem plantas de pequenas dimensões, por causa de suas pequenas folhas e outras partes que liberam aromas tornando-as essenciais para a culinária e outros usos domésticos. Essas plantas têm como função desempenhar um grande papel de interesse para as pesquisas cientificas por causa de seus metabólitos primários e secundários (Sales,2015).

Desde então, seu poder curativo foi evidenciado pelas primeiras civilizações. Onde detectando que tais plantas tinham suas próprias essências, seus princípios ativos, que quando experimentados poderiam ser uteis para combater algumas situações patológicas. Assim, o conhecimento tradicional ao longo das gerações, foram passados, manipulados e utilizados para as mais diversificadas atividades terapêuticas (Silva et al., 2018).

Em relação a biodiversidade, o Brasil é reconhecido por possuir uma abundância destas plantas e seus recursos naturais, inclusive na Amazônia, uma região que está relacionada diretamente com os povos e comunidades tradicionais. Onde a fitoterapia vem desempenhar um papel fundamental para valorizar e resgatar os costumes em relação ao uso seguro e racional de plantas medicinais em prol da saúde e bem-estar da população (Farias et al., 2019).

As principais famílias de plantas aromáticas que predominam na região amazônica são (em ordem decrescente): Piperaceae, Asteraceae, Myrtaceae, Lamiaceae, Annonaceae, Lauraceae, Euphorbiaceae, Verbenaceae, Scrophulariaceae, 
Anacardiaceae. Existem diversas espécies de plantas aromáticas que podem ser cultivadas, por esse motivo, 10 espécies destas plantas foram selecionadas mediante pesquisa e revisão bibliográfica. Com as seguintes informações: Nome cientifico; nome popular; partes utilizadas; propriedade farmacológica; indicação terapêutica, conforme mostra o Quadro 1.

Quadro 1: Plantas selecionadas para o estudo mediante revisão bibliográfica, apresentando as principais informações em relação ao uso seguro dessas plantas medicinais para fitoterapia.

\begin{tabular}{|c|c|c|c|c|c|}
\hline $\begin{array}{c}\text { NOME } \\
\text { POPULAR }\end{array}$ & $\begin{array}{c}\text { NOME } \\
\text { CIENTÍFICO }\end{array}$ & $\begin{array}{l}\text { RTES } \\
\text { IZADAS }\end{array}$ & $\begin{array}{c}\text { PROPRIEDADE } \\
\text { FARMACOLÓGICA }\end{array}$ & $\begin{array}{c}\text { INDICAÇÃO } \\
\text { TERAPÊUTICA }\end{array}$ & REFERÊNCIAS \\
\hline Alecrim & Rosmarinus officinalis & $\begin{array}{l}\text { Folhas e } \\
\text { flores. }\end{array}$ & $\begin{array}{c}\text { Anti-inflamatória; } \\
\text { antioxidante; } \\
\text { antiespasmódicas; } \\
\text { antimicrobiana; } \\
\text { antifúngica. }\end{array}$ & $\begin{array}{l}\text { Dor de estomago; } \\
\text { dor reumática; } \\
\text { cicatrizante; } \\
\text { enxaqueca; } \\
\text { antialérgico } \\
\end{array}$ & $\begin{array}{l}\text { Gonçalves et al., } 2017 . \\
\text { Melo et al., } 2021 .\end{array}$ \\
\hline Sálvia & Salvia officinalis & $\begin{array}{l}\text { Folhas e } \\
\text { flores }\end{array}$ & $\begin{array}{l}\text { Ação digestiva; anti- } \\
\text { inflamatória; } \\
\text { hipoglicemiante; } \\
\text { antimicrobiana; } \\
\text { bactericida }\end{array}$ & $\begin{array}{c}\text { Ansiedade; } \\
\text { cicatrizante; redutor } \\
\text { de lactação; } \\
\text { leucorreia; feridas; } \\
\text { glicose; }\end{array}$ & $\begin{array}{l}\text { Zeni et al., } 2016 . \\
\text { Sales, } 2015 .\end{array}$ \\
\hline Capim-limão & Cymbopogon citratus & $\begin{array}{l}\text { Folhas, } \\
\text { rizomas e } \\
\text { raízes } \\
\text { frescas } \\
\text { ou secas }\end{array}$ & $\begin{array}{l}\text { Antiespasmódicos; } \\
\text { antimicrobiana; } \\
\text { antibacteriana; } \\
\text { antirreumático; } \\
\text { calmante }\end{array}$ & $\begin{array}{l}\text { Resfriados; dor de } \\
\text { cabeça; ansiedade; } \\
\text { asma; cólicas }\end{array}$ & $\begin{array}{c}\text { Borges \& Sales, } 2018 . \\
\text { Silva et al., } 2018 .\end{array}$ \\
\hline Erva-cidreira & Melissa officinalis & Folhas & $\begin{array}{c}\text { Anti-inflamatório; } \\
\text { antibacteriano; } \\
\text { antiasmática; sedativa; } \\
\text { tônica; carminativa; } \\
\text { calmante } \\
\end{array}$ & $\begin{array}{l}\text { Asma; náuseas; } \\
\text { cefaleias; insônia; } \\
\text { bronquite crônica; } \\
\text { gripe; crises } \\
\text { nervosas } \\
\end{array}$ & $\begin{array}{l}\text { Bortuluzzi, Schmitt \& } \\
\text { Mazur, } 2019 . \\
\text { Araújo, } 2015 .\end{array}$ \\
\hline Hortelã comum & Mentha spicata & $\begin{array}{l}\text { Folhas; } \\
\text { flores }\end{array}$ & $\begin{array}{c}\text { Antioxidante; } \\
\text { antiespasmódica; } \\
\text { carminativa; calmante }\end{array}$ & $\begin{array}{l}\text { Cólicas intestinais; } \\
\text { sinusite; flatulências; }\end{array}$ & $\begin{array}{c}\text { Lins, } 2015 . \\
\text { Ibrahim, } 2017\end{array}$ \\
\hline Alfavaca & Ocimum gratissimum $L$ & $\begin{array}{l}\text { Folhas e } \\
\text { flores }\end{array}$ & $\begin{array}{c}\text { Antifúngico; } \\
\text { antibacteriano; anti- } \\
\text { inflamatória; }\end{array}$ & $\begin{array}{l}\text { Cólicas menstruais; } \\
\text { cefaleias; mal-estar; } \\
\text { infecção urinária. }\end{array}$ & $\begin{array}{l}\text { Rocha et al., } 2020 . \\
\text { Farias et al., } 2019 .\end{array}$ \\
\hline Alfazema & Lavandula angustifólia & $\begin{array}{l}\text { Folhas e } \\
\text { flores }\end{array}$ & $\begin{array}{l}\text { Antiespasmódica; } \\
\text { sedativa; } \\
\text { anticonvulsivante; } \\
\text { antifúngica; } \\
\text { antioxidante; anti- } \\
\text { inflamatória. }\end{array}$ & $\begin{array}{l}\text { Tosse; enxaqueca; } \\
\text { tensão muscular; } \\
\text { gripe; sinusite; } \\
\text { insônia; }\end{array}$ & $\begin{array}{l}\text { Lima et al., } 2021 . \\
\text { Adamuchio, } 2017 .\end{array}$ \\
\hline Boldo brasileiro & Plectranthus barbatus & $\begin{array}{l}\text { Folhas; } \\
\text { caule e } \\
\text { raiz }\end{array}$ & $\begin{array}{l}\text { Anti-inflamatória; } \\
\text { antioxidante; } \\
\text { antimicrobiana; } \\
\text { antifúngica; } \\
\text { hipoglicemica; } \\
\text { antipirética. }\end{array}$ & $\begin{array}{c}\text { Câncer; HIV; } \\
\text { distúrbios } \\
\text { digestivos; } \\
\text { hepáticos; glicemia }\end{array}$ & $\begin{array}{l}\text { Monthana et al., } 2019 . \\
\text { Rosal, 2021. } \\
\text { Kapewangolo \& Meyer, } \\
2018 .\end{array}$ \\
\hline Orégano & Origanum vulgare $L$ & & $\begin{array}{l}\text { Antiespasmódico; } \\
\text { antisséptico; } \\
\text { carminativa; anti- } \\
\text { inflamatória; } \\
\text { antimicrobiana; } \\
\text { antimalárico. } \\
\end{array}$ & $\begin{array}{c}\text { Asma; bronquite; } \\
\text { perda de apetite; } \\
\text { flatulências; } \\
\text { distúrbios } \\
\text { gastrointestinais; } \\
\text { tosse; dor de cabeça. }\end{array}$ & $\begin{array}{l}\text { Marreiros et al., } 2015 . \\
\text { Sakurai et al., } 2016 .\end{array}$ \\
\hline Arruda & Ruta graveolens $L$ & $\begin{array}{l}\text { Folhas e } \\
\text { flores, } \\
\text { raízes, } \\
\text { caules }\end{array}$ & $\begin{array}{l}\text { Anti-helmíntica; } \\
\text { abortiva; carminativa; } \\
\text { antiespasmódica; } \\
\text { estimulante; anti- } \\
\text { hemorrágica. }\end{array}$ & $\begin{array}{l}\text { Reumatismos; } \\
\text { verminoses; gota; } \\
\text { hipertensão; } \\
\text { afecções de rins, } \\
\text { fígado; dor de dente } \\
\text { e ouvido. }\end{array}$ & $\begin{array}{l}\text { Lima, } 2018 . \\
\text { Cruz, } 2017 .\end{array}$ \\
\hline
\end{tabular}

Fonte: Autores.

Como observado no Quadro 1, a utilização das plantas aromáticas ou medicinais são bastante utilizadas para o tratamento de doenças das mais variadas etiologias, na qual busca valorizar uma prática tradicional que está enraizada na cultura local, acredita-se que devido as condições econômicas e a falta de assistência médica, o uso destas plantas medicinais é um fator 
determinante para a contribuição da utilização destes recursos vegetais para a finalidade terapêutica e fortalecimento da atenção básica.

$\mathrm{Na}$ literatura científica, encontram-se registros de muitas espécies vegetais endêmicas da região amazônica com a utilização das plantas aromáticas no tratamento de doenças. Como resultado tem-se uma crescente necessidade de estudos para obtenção de mais informações e esclarecimentos sobre os efeitos terapêuticos e da ação farmacológica que estas plantas podem causar no organismo. Nesta região, podem ser encontradas variedades de plantas aromáticas com propriedades analgésicas, adstringentes, tônicos, anti-inflamatórios, antidiabéticos e carminativa que são amplamente usados pela população local por exemplo (Marreiros et al., 2015).

Na agricultura em relação a elevada demanda do cultivo de plantas aromáticas houve um crescimento na geração de empregos aos produtores rurais que passaram a ter essa atividade como um forte apelo econômico e social de desenvolvimento. Em geral, o mercado deste segmento, além de ser bastante específico, seu cultivo é muito amplo, podendo ser utilizadas para cultivos de ervanários, farmácias de manipulação, laboratórios farmacêuticos, atacadistas, programas de saúde das Prefeituras e das pastorais de saúde dos hospitais de medicina alternativa e ainda utilizadas para pesquisas cientificas (Senar,2017).

A utilização e consumo de plantas aromáticas é reconhecida atualmente pela Organização Mundial da Saúde - OMS, devido ao conhecimento empírico secular sobre a ação desses vegetais reconhecido por diversos grupos étnicos, um fato que resultou na descoberta da medicina tradicional. No Brasil, além dos conhecimentos tradicionais indígenas, as contribuições trazidas de outros povos também tiveram uma importância significativa no surgimento da medicina popular rica baseada na utilização da biodiversidade de vegetais nacionais, promovendo uma infinidade de diferentes espécies de plantas medicinais em prol à saúde humana (Borges \& Sales, 2018).

A exemplo das plantas medicinais, o crescimento do mercado de plantas aromáticas cresceu devido ao aumento de buscas por corantes e aromatizantes naturais pela indústria de alimentos e bebidas, e na expansão da indústria de cosméticos e perfumes, no qual destaca-se, o comércio de óleos essenciais como matéria-prima para essas indústrias.

Exemplificando, as espécies destas plantas aromáticas mediante revisão bibliográfica de acordo com sua finalidade de uso terapêutico podemos citar por exemplo:

$>$ Alecrim (Rosmarinus officinalis): condimentar, aromático e medicinal;

$>$ Sálvia (Salvia officinalis): condimentar e medicinal;

$>$ Capim-santo (Cymbopogon citratus): medicinal, aromático e condimentar;

$>$ Erva-cidreira (Melissa officinalis): medicinal e aromático;

$>$ Hortelã comum (Mentha spicata): condimentar e medicinal;

$>$ Alfavaca (Ocimum gratissimum L): condimentar, medicinal e aromático;

$>$ Alfazema (Lavandula angustifólia): aromático, medicinal;

$>$ Boldo brasileiro (Plectranthus barbatus): medicinal;

$>$ Orégano (Origanum vulgare $L)$ : aromático, medicinal e condimentar;

$>$ Arruda (Ruta graveolens L): medicinal e cosmético.

A implementação da fitoterapia no âmbito dos serviços de Atenção Básica à Saúde (ABS) no Brasil teve início em 1988, principalmente após a descentralização, incentivo da participação popular e crescimento da autonomia municipal na área da saúde (Gouveia \& Simionato, 2019). Por esse motivo, o Ministério da Saúde, propôs o objetivo de ampliar o acesso da população aos serviços relacionados a fitoterapia, aprovando a Política Nacional de Práticas Integrativas e Complementares (PNPIC) em Saúde por meio da Portaria nº 971 , de 03 de maio de 2006 e a Política Nacional de Plantas Medicinais e Fitoterápico (PNPMF) pelo Decreto 5813, 26 de junho de 2006 (Brasil, 2018). 
Por meio da Farmacopeia Brasileira, em novembro de 2011, a ANVISA lançou o Formulário de Fitoterápicos da Farmacopeia Brasileira (Resolução RDC n 60 de 10 de novembro de 2011), voltado principalmente para as práticas de manipulação e dispensação de fitoterápicos, contribuindo com os Serviços de Fitoterapia e Farmácias Vivas existentes em todo o país. Após, o sucesso da publicação da $1^{\mathrm{a}}$ edição houveram novas discussões a respeito dos fitoterápicos, que a partir disso, foi criada uma classe de Produto Tradicional fitoterápico de acordo com a nova Resolução RDC nº 26 de 13 de maio de 2014, baseado conforme a notificação seguindo o Formulário de Fitoterápicos da Farmacopeia Brasileira (Brasil, 2018).

Assim, de acordo com a pesquisa as plantas aromáticas que estão incluídas neste formulário de fitoterápicos RDC nº 26 de 13 de maio de 2014, foram 4 espécies: Alecrim (Rosmarinus officinalis), Boldo brasileiro (Plectranthus barbatus), Capimsanto (Cymbopogon citratus) e Erva-cidreira (Melissa officinalis), apresentaram todas as informações necessárias desde a identificação da espécie até as principais classes químicas composta em cada parte utilizada da planta. Por fim em 2016, a ANVISA publicou o Memento Fitoterápico, uma manual de prescrição de medicamentos fitoterápicos que tem como principal finalidade de padronização e estimulo da fitoterapia clinica no Brasil, modulando dessa forma como os profissionais devem prescrever e como eles devem proceder frente a interações medicamentosas, efeitos adversos, orientações acerca do uso e muito mais estão disponíveis nesse documento.

Por tanto a fitoterapia e o uso de plantas medicinais são de grande importância quando relacionada ao tratamento de pacientes ou consumidores de menor renda que atribuem o seu uso na prática da medicina popular. Pois, a fitoterapia é uma das práticas terapeuticas mais antigas utilizadas pela população, sendo assim, um recurso de prevenção e tratamento de patologias em que utiliza partes destas plantas ou plantas inteiras para benefícios curativos, paliativos à saúde do homem. Entretanto, o consumo excessivo ou o mal uso destas plantas pode provocar reações alérgicas, efeitos sinérgicos negativos ou efeitos adversos que podem gerar quadro de intoxicações agudas por exemplo. Principalmente devido que algumas plantas retêm princípios ativos com efeitos desconhecidos ou até tóxicos (como por exemplo os compostos cinogenicos). Por esse motivo, a fitoterapia é um tratamento caracterizado pelo uso de plantas medicinais que utiliza substâncias ativas na forma de fitocomplexos, onde um marcador químico desempenhará o papel de controle de qualidade, dessa forma garantindo integridade química e farmacológica do material (Rocha et al, 2020).

O emprego da fitoterapia à Atenção Primária de saúde se mostrou um fator importante na contribuição do resgate de conhecimentos populares e culturais contribuindo para o aumento do vínculo entre os pacientes e à equipe de saúde permitindo que este seja um agente ativo em seu processo de saúde/adoecimento. Dessa forma, a medicina complementar e alternativa utilizam destas principais ferramentas que estão relacionadas a fitoterapia e utilização das plantas medicinais, pelo fato que a população já desfruta destes benefícios desde os primórdios em suas famílias ou comunidades, utilizando os vários tipos de plantas em seus tratamentos de saúde, através da medicina tradicional/popular ou dos programas inseridos dentro do SUS (Lima, 2018).

Por tanto trabalhos que busquem sistematizar o uso correto, racional e integrando o conhecimento tradicional ao conhecimento cientifico, são fundamentais para a fixação da fitoterapia como modelo de promoção de saúde bem como fornecer a população segurança no uso desses ativos para a promoção de sua saúde e bem-estar.

\section{Conclusão}

A utilização de plantas aromáticas e também de plantas medicinais para o tratamento de doenças é uma prática bastante enraizada na cultura da região amazônica, mostrando que apesar do conhecimento e entendimento em relação ao efeito farmacológico que cada tipo de plantas pode provocar a saúde, ainda é pouco estudada na questão dos princípios ativos presentes em cada parte usada destas plantas medicinais. 
Nosso estudo identificou dentro da fitoterapia e da medicinal tradicional, diferentes abordagens e costumes acerca do uso correto no manejo de plantas medicinais. Além de contribuir para difundir o conhecimento a cerca dessa classe de plantas (aromáticas), nosso estudo vem a fornecer ferramentas com base na leitura acerca da forma do uso para o preparo mais eficiente dessas espécies aqui revisadas.

Sendo assim, é de grande importância para a comunidade científica que se continue a investigar e estudar as propriedades terapêuticas das plantas aromáticas e dessa forma possamos oferecer mais opções de tratamento acessíveis para a população, contribuindo de forma direta ou indireta para o fortalecimento e manutenção da fitoterapia e o conhecimento tradicional acerca do uso seguro e racional de de plantas medicinais.

\section{Referências}

Adamuchio, L. G. I. Deschamps, C. \& Machado, M. P. (2017). Aspectos gerais sobre a cultura da Lavanda (Lavandula spp.) Rev. Bras. Pl. Med., 19(4), 483490.

Araújo, M. S. C., Costa, J. W., Costa, A. A., Tocchio, P. S. P. L., Araújo, L. S. A. \& Nunes, V. M. A. (2015). A utilização de plantas medicinais e da fitoterapia em comunidades assistidas pela estratégia saúde da família. Revista Brasileira Pesquisa em Saúde. 17(4): 6-16.

Bortoluzzi, M. M.; Schmitt, V.; Mazur, C. E. (2019). Efeito fitoterápico de plantas medicinais sobre a ansiedade: uma breve revisão. Revista Research, Society and Development, 9(1), e02911504.

Borges, F. V. \& Sales, M. D. C. (2018). Políticas públicas de plantas medicinais e fitoterápicos no Brasil: sua história no sistema de saúde. Pensar Acadêmico. 16(1): 13-27.

Brasil. Agência Nacional de Vigilância Sanitária (2018). Formulário de Fitoterápico Farmacopeia Brasileira. Anvisa. 160 p. https:/www.anvisa.gov.br/ hotsite /fa rmac opeiabrasileira/conteúdo/Formulario_de_Fitoterapicos_da_Farmacopeia_Brasileira.pdf.

Correia Junior, C., Lin, C. M. \& Scheffer, M. C. (2014). Cultivo de Plantas Medicinais, Condimentares e Aromáticas. FUNEP.

Cruz, M. J. F. \& Bezerra, S. B. (2018). Obtenção do óleo essencial de Ocimum gratissimum, L para desenvolvimento de cosmético de limpeza facial. Revista Diálogos Acadêmicos, 6(2), 115-122

Cruz, S. P. de A. (2017). Abordagem Bibliográfica sobre os riscos da utilização da Arruda (Ruta graveolens L) na gestação. FAEMA. Monografia, Arquimedes.

Farias, J. C. et al. (2019). Medicinal flora cultivated in backyards of a community in Northeast Brazil. Ethnobotany Research and Applications, $18,1-13$.

Ferreira, et al. (2017). Caracterização do perfil fenólico do extrato aquoso e hidroetanólico de Rosmarinus officinalis L. (Chemical characterization of the Rosmarinus officinalis L. aqueous and hydroethanolic extracts in terms of phenolic compounds). Revista SCALP (Sociedade de Ciências Agrárias de Portugal), 40(especial): 147-150. http://dx.doi.org/10.19084/RCA16232.

Gonçalves, et al. (2017). Plantas Medicinais: Relacionando conhecimento popular e cientifico na Atenção Primária à Saúde. Revista Visão Acadêmica. 18(4), 41.

Ianck, M. A. (2017). Conhecimento e uso de plantas medicinais por usuários de unidades básicas de saúde na região de Colombo-PR. Revista Saúde e Desenvolvimento, 11(8), 29-30.

Ibrahim, H. M. (2017). Evaluation of genetic diversity and relationships of five Mentha species using RAPD markers. Journal of Current Science. 6, $271-27$.

Kapewangolo, P. \& Meyer, D. (2018). Plectranthus barbatus; Antioxidant, and Other Inhibitory Responses Against HIV/AIDS. In: PREEDY, V. R.; WATSON, R. R. (eds). HIV/AIDS: Oxidative Stress and Dietary Antioxidants, 149-159.

Lima, et al. (2021). A utilização de óleos essenciais de Lavandula angustifólia, Pelargonium graveolens e Citrus bergamia no combate à ansiedade. Revista Brazilian Journal of Development. 10.34117/bjdv7n4-525.

Lima, A. K. R. (2018). Estudo de Prospecção Científica Tecnológica da Atividade medicinal da espécie Ruta graveolens (ARRUDA). 44 p. Monografia (Graduação). Curso de Ciências Naturais Biologia, Universidade Federal de Maranhão, Pinheiro.

Lins, A. D. F. et al. (2015). Quantificação de Compostos Bioativos em Erva Cidreira (Melissa offcinalis L.) e Capim Cidreira (Cymbopogon citratus Stapf). Revista da UFPB - Gaia Scientia, 17-21.

Marreiros, P. M., Pinto, B. L. S. \& Nascimento, V. T. (2015). Can organoleptic properties explain the differential use of medicinal plants Evidence from Northeastern Brazil. Journal of Ethnopharmacology, 159,43-48.

Melo, et al. (2021). Alecrim (Rosmarinus officinalis L.) Atividade anti-inflamatória: uma revisão de literatura. Revista de Casos e Consultoria, 12 (1), e24346. ISSN 22377417.

Monthana, R. A. et al. (2019). Comparative evaluation of cytotoxic, antimicrobial and antioxidant activities of the crude extracts of three Plectranthus species grown in Saudi Arabia, Saudi Pharmaceutical Journal, 27(2), 162-170. 
Research, Society and Development, v. 10, n. 14, e419101422263, 2021

(CC BY 4.0) | ISSN 2525-3409 | DOI: http://dx.doi.org/10.33448/rsd-v10i14.22263

Rocha, V. L. P. et al. (2020). Anatomia comparada, histoquímica e fitoquímica dos órgãos vegetativos de espécies do gênero Ocimum L. (Lamiaceae). Revista Ibero-Americana de Ciências Ambientais, 11(2), 266-277.

Rodrigues, G. S. Santos, N. O. \& Fortuna, J. L. (2020). Atividade antimicrobiana do óleo essencial de Cymbopogon citratus (D. C.) Stapf. (Capim-Santo) sobre Staphylococcus aureus e. Escherichia coli. Revista Ciência, Tecnologia e Ambiente, 10, e 10177.

Rosal, G. B. (2021). Desenvolvimento e Produtividade do boldo brasileiro submetido a lâminas de irrigação. 59 fls.: in. Color. R698d. Dissertação (mestrado) - Universidade Federal do Ceará, centro de Ciências Agrárias, Programa de Pós-Graduação em Engenharia Agrícola. Fortaleza.

Sales, H. J. S. P. (2015). Revisão: Lavandula L.- Aplicação da cultura in vitro à produção de óleos essenciais e seu potencial econômico em Portugal. Rev. Bras. Pl. Med., 17(4), 992-999.

Sakurai, F. N. et al. (2016). Caracterização das propriedades funcionais das ervas aromáticas utilizadas em um hospital especializado em cardiopneumologia. Demetra, 11(4), 1097-1113.

Senar, (2017). Plantas medicinais aromáticas e condimentares: produção e beneficiamento / Serviço Nacional de Aprendizagem Rural. 124.

Silva, L. F. L. et al. (2018). Nutritional evaluation of non-conventional vegetables in Brazil. Anais da Academia Brasileira de Ciências, 90(2), $1775-1787$.

Zeni, A. L. B., Parisotto, A. V., Mattos, G. \& Helena, E. T. S. Utilização de plantas medicinais como remédio caseiro na atenção primária em Blumenau, Santa Catarina, Brasil. Revista Ciência \& Saúde Coletiva. 22(8): 2703-2712. 